\title{
Maximal 0-1-fillings of moon polyominoes with restricted chain lengths and rc-graphs
}

\author{
Martin Rubey $\|^{t}$ \\ Institut für Algebra, Zahlentheorie und Diskrete Mathematik, Leibniz Universität Hannover, Hannover, Germany
}

\begin{abstract}
We show that maximal 0-1-fillings of moon polynomials, with restricted chain lengths, can be identified with certain rc-graphs, also known as pipe dreams. In particular, this exhibits a connection between maximal 0-1fillings of Ferrers shapes and Schubert polynomials. Moreover, it entails a bijective proof showing that the number of maximal fillings of a stack polyomino $S$ with no north-east chains longer than $k$ depends only on $k$ and the multiset of column heights of $S$.

Our main contribution is a slightly stronger theorem, which in turn leads us to conjecture that the poset of rc-graphs with covering relation given by generalised chute moves is in fact a lattice.

Résumé. Nous démontrons que les remplissages maximaux avec 0 et 1 des polyominos $L$-convexes, avec longueurs de chaînes restreintes, peuvent être identifiés avec certains $r$-graphes, également connus sous le nom de pipe dreams. En particulier, ceci montre un lien entre ces remplissages d'un diagramme de Ferrers et les polynômes de Schubert. On en déduit en outre une preuve bijective du fait que le nombre de remplissages maximaux d'un stack polyomino $S$, avec longueurs de chaînes bornées par un entier $k$, dépend seulement de $k$ et du multi-ensemble des tailles des colonnes de $S$.
\end{abstract}

Notre contribution principale est un énoncé un peu plus fort, qui nous mène à conjecturer que l'ensemble ordonné (poset) des rc-graphes est en fait un treillis.

Keywords: multitriangulations, rc-graphs, Edelman-Greene insertion, Schubert polynomials

\section{Introduction}

\subsection{Triangulations, multitriangulations and 0-1-fillings}

The systematic study of 0-1-fillings of polyominoes with restricted chain lengths likely originates in an article by Jakob Jonsson [5]. At first, he was interested in a generalisation of triangulations, where the objects under consideration are maximal sets of diagonals of the $n$-gon, such that at most $k$ diagonals are allowed to cross mutually. Thus, in the case $k=1$ one recovers ordinary triangulations. He realised these objects as fillings of the staircase shaped polyomino with row-lengths $n-1, n-2, \ldots, 1$ with zeros and ones. The condition that at most $k$ diagonals cross mutually then translates into the condition that

†martin.rubey@math.uni-hannover. de

1365-8050 @ 2011 Discrete Mathematics and Theoretical Computer Science (DMTCS), Nancy, France 
the longest north-east chain in the filling has length $k$, see Definition 2.3 Instead of studying fillings of the staircase shape only, he went on to consider more general shapes which he called stack and moon polyominoes, see Definition 2.2 and Figure 1 .

For stack polyominoes he was able to prove that the number of maximal fillings depends only on $k$ and the multiset of heights of the columns, not on the particular shape of the polyomino. He conjectured that this statement holds more generally for moon polyominoes, which was eventually proved by the author [13] using a technique introduced by Christian Krattenthaler [8] based on Sergey Fomin's growth diagrams for the Robinson-Schensted-Knuth correspondence. However, the proof given there is not fully bijective: what one would hope for is a correspondence between fillings of any two moon polyominoes that differ only by a permutation of the columns. This article is a step towards this goal.

\subsection{RC-graphs and the subword complex}

RC-graphs (for 'reduced word compatible sequence graphs', see [1], also known as 'pipe dreams' see [7]) were introduced by Sergey Fomin and Anatol Kirillov [3] to prove various properties of Schubert polynomials. Namely, for a given permutation $w$, the Schubert polynomial $\mathfrak{S}_{w}$ can be regarded as the generating function of rc-graphs, see the remark after Definition 2.5

A different point of view is to consider them as facets of a certain simplicial complex. Let $w_{0}$ be the long permutation $n \cdots 21$, and consider its reduced factorisation

$$
Q=s_{n-1} \cdots s_{2} s_{1} s_{n-1} \cdots s_{3} s_{2} \cdots \cdots s_{n-1} s_{n-2} s_{n-1} .
$$

Then the subword complex associated to $Q$ and $w$ introduced by Allen Knutson and Ezra Miller [7, 6] has as facets those subwords of $Q$ that are reduced factorisations of $w$. Subword complexes enjoy beautiful topological properties, which are transferred by the main theorem of this article to the simplicial complex of 0-1-fillings, as observed by Christian Stump [17], see also the article by Luis Serrano and Christian Stump [15].

The intimate connection between maximal fillings and rc-graphs demonstrated by the main theorem of this article, Theorem 3.2, should not have come as a surprise. Indeed, Sergey Fomin and Anatol Kirillov [4] established a connection between reduced words and reverse plane partitions already thirteen years ago, which is not much less than the case of Ferrers shapes in Theorem 4.3. They even pointed towards the possibility of a bijective proof using the Edelman-Greene correspondence.

More recently, the connection between Schubert polynomials and triangulations was noticed by Alexander Woo [18]. Vincent Pilaud and Michel Pocchiola [11] discovered rc-graphs (under the name 'beam arrangements') more generally for multitriangulations, however, they were unaware of the theory of Schubert polynomials. In particular, Theorem 3.18 of Vincent Pilaud's thesis [10] (see also Theorem 21 of [11]) is a variant of our Theorem 3.2 for multitriangulations.

Finally, Christian Stump and the author of the present article became aware of an article by Vincent Pilaud and Francisco Santos [12] that describes the structure of multitriangulations in terms of so-called $k$-stars (introduced by Harold Coxeter). We then decided to translate this concept to the language of fillings, and discovered pipe dreams yet again. 

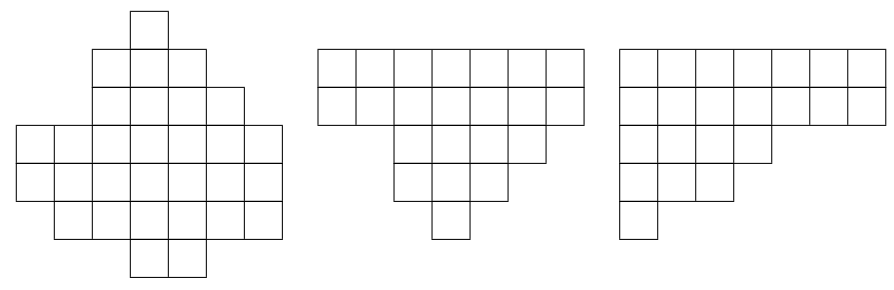

Fig. 1: a moon-polyomino, a stack-polyomino and a Ferrers diagram

\section{Definitions}

\subsection{Polyominoes}

Definition 2.1 A polyomino is a finite subset of the quarter plane $\mathbb{N}^{2}$, where we regard an element of $\mathbb{N}^{2}$ as a cell. A column of a polyomino is the set of cells along a vertical line, a row is the set of cells along a horizontal line. We are using 'English' (or matrix) conventions for the indexing of the rows and columns of polyominoes: the top row and the left-most column have index 1.

The polyomino is convex, if for any two cells in a column (rsp. row), the elements of $\mathbb{N}^{2}$ in between are also cells of the polyomino. It is intersection-free, if any two columns are comparable, i.e., the set of row coordinates of cells in one column is contained in the set of row coordinates of cells in the other. Equivalently, it is intersection-free, if any two rows are comparable.

For example, the polyomino

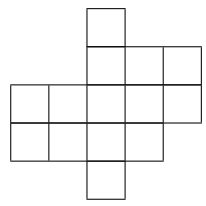

is convex, but not intersection-free, since the first and the last columns are incomparable.

Definition 2.2 A moon polyomino (or L-convex polyomino) is a convex, intersection-free polyomino. Equivalently we can require that any two cells of the polyomino can be connected by a path consisting of neighbouring cells in the polyomino, that changes direction at most once. A stack polyomino is a moonpolyomino where all columns start at the same level. A Ferrers diagram is a stack-polyomino with weakly decreasing row widths $\lambda_{1}, \lambda_{2}, \ldots, \lambda_{n}$, reading rows from top to bottom.

Because a moon-polyomino is intersection free, the set of rows of maximal length in a moon polyomino must be consecutive. We call the set of rows including these and the rows above the top half of the polyomino. Similarly, the set of columns of maximal length, and all columns to the right of these, is the right half of the polyomino. The intersection of the top and the right half is the top right quarter of $M$.

\subsection{Fillings and Chains}

Definition 2.3 A 0-1-filling of a polyomino is an assignment of the numbers 0 and 1 to the cells of the polyomino. Cells containing 0 are also called empty.

A north-east chain is a sequence of non-zero entries in a filling such that the smallest rectangle containing all its elements is completely contained in the moon polyomino and such that for any two of its elements one is strictly to the right and strictly above the other. 

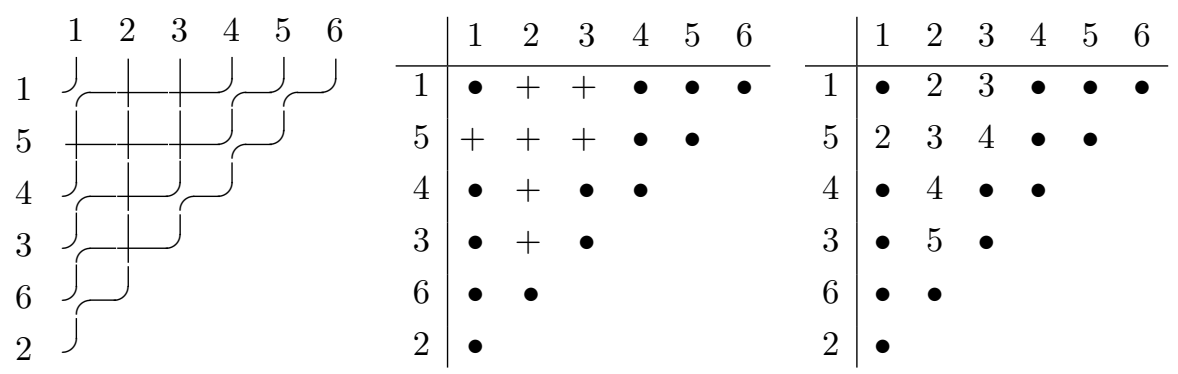

Fig. 2: a reduced pipe dream associated to the reduced factorisation $s_{3} s_{2} s_{4} s_{3} s_{2} s_{4} s_{5}$ of 1, 5, 4, 3, 6, 2 .

As it turns out, it is more convenient to draw dots instead of ones and leave cells filled with zeros empty. Two examples of (rather special) fillings of a moon polyomino are depicted in Figure 4

Definition 2.4 $\mathcal{F}_{01}^{n e}(M, k)$ is the set of 0-1-fillings of the moon polyomino $M$ whose longest north-east chain has length $k$ and that are maximal, i.e., assigning an empty cell a 1 would create a north-east chain of length $k+1$. For a vector $\mathbf{r}$ of integers $\mathcal{F}_{01}^{n e}(M, k, \mathbf{r})$ is the subset of $\mathcal{F}_{01}^{n e}(M, k)$ consisting of those fillings that have exactly $\mathbf{r}_{i}$ zero entries in row $i$.

For any filling in $\mathcal{F}_{01}^{n e}(M, k)$, and an empty cell $\epsilon$, there must be a chain $C$ such that replacing the 0 with 1 in $\epsilon$, and adding $\epsilon$ to $C$, would make $C$ into a $(k+1)$-chain. In this situation, we say that $C$ is a maximal chain for $\epsilon$.

Note that extending the first $k$ rows and columns of a Ferrers diagram does not affect the set $\mathcal{F}_{01}^{n e}$, which is why we choose to fix the number of zero entries instead of entries equal to 1 , which might seem more natural at first glance.

For the staircase shape $\lambda_{0}=(n-1, \ldots, 1)$, the set $\mathcal{F}_{01}^{n e}\left(\lambda_{0}, k\right)$ has a particularly beautiful interpretation, namely as the set of $k$-triangulations of the $n$-gon. More precisely, label the vertices of the $n$-gon clockwise from 1 to $n$, and identify a cell of the shape in row $i$ and column $j$ with the pair $(n-i+1, j)$ of vertices. Thus, the entries in the filling equal to 1 define a set of diagonals of the $n$-gon. It is not hard to check that a north-east chain of length $k$ in the filling corresponds to a set of $k$ mutually crossing diagonals in the $n$-gon. This correspondence was Jakob Jonsson's [5] starting point to prove (in a quite non-bijective fashion) that there are as many $k$-triangulations of the $n$-gon as fans of $k$ non-intersecting Dyck paths with $n-2 k$ up steps each. For this case Luis Serrano and Christian Stump [15] provided the first completely bijective proof.

\subsection{Pipe dreams}

In this section we collect some results around rc-graphs. All of these can be found in [1] together with precise references.

Definition 2.5 A pipe dream for a permutation $w$ is a filling of a the quarter plane $\mathbb{N}^{2}$, regarding each element of $\mathbb{N}^{2}$ as a cell, elbow joints $-\sim$ and a finite number of crosses + , such that a pipe entering from above in column $i$ exits to the left from row $w^{-1}(i)$. A pipe dream is reduced if each pair of pipes 
crosses at most once, it is then also called rc-graph. $\mathcal{R C}(w)$ is the set of reduced pipe dreams for $w$, and $\mathcal{R C}(w, \mathbf{r})$ is the subset of $\mathcal{R C}(w)$ having precisely $\mathbf{r}_{i}$ crosses in row $i$.

Usually it will be more convenient to draw dots instead of elbow joints and sometimes to omit crosses. We will do so without further notice.

Every pipe dream in $\mathcal{R C}(w)$ is associated to a reduced factorisation of $w$ as follows: replace each cross appearing in row $i$ and column $j$ of the pipe dream with the elementary transposition $(i+j-1, i+j)$. Then the reduced factorisation of $w$ is given by the sequence of transpositions obtained by reading each row of the pipe dream from right to left, and the rows from top to bottom. Figure 2 shows an example.

Using reduced pipe dreams, it is possible to define the Schubert polynomial $\mathfrak{S}_{w}$ for the permutation $w$ in a very concrete way. For a reduced pipe dream $D \in \mathcal{R C}(w)$, define $x^{D}=\prod_{(i, j) \in D} x_{i}$, where the product runs over all crosses in the pipe dream. Then the Schubert polynomial is just the generating function for pipe dreams:

$$
\mathfrak{S}_{w}=\sum_{D \in \mathcal{R C}(w)} x^{D}
$$

The following operation on pipe dreams, in a slightly less general form, was introduced by Nantel Bergeron and Sara Billey [1]. It will be the main tool in the proof of Theorem 3.2.

Definition 2.6 Let $D \in \mathcal{R C}(w)$ be a pipe dream. Then a chute move is a modification of $D$ of the following form:
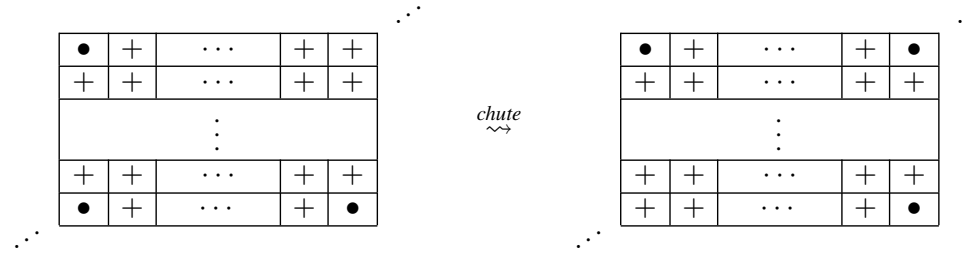

More formally, a chutable rectangle is a rectangular region $r$ inside a pipe dream $D$ with at least two columns and two rows such that all but the following three locations of $r$ are crosses: the north-west, south-west, and south-east corners. Applying a chute move to $D$ is accomplished by placing $a$ '+' in the south-west corner of a chutable rectangle $r$ and removing the '+' from the north-east corner of $r$. We call the inverse operation inverse chute move.

The following lemma was given by Nantel Bergeron and Sara Billey [1, Lemma 3.5] for two rowed chute moves, the proof is valid for our generalised chute moves without modification:

Lemma 2.7 The set $\mathcal{R C}(w)$ of reduced pipe dreams for $w$ is closed under chute moves.

Proof: The pictorial description of chute moves immediately implies that the permutation associated to the pipe dream remains unchanged. For example, here is the picture associated with a two rowed chute move:
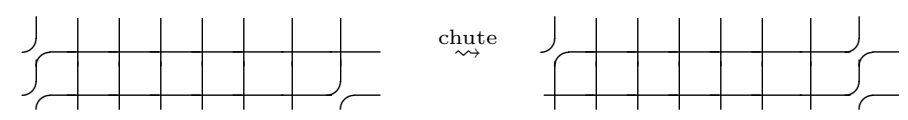
It follows that chute moves define a partial order on $\mathcal{R C}(w)$, where $D$ is covered by $E$ if there is a chute move transforming $E$ into $D$. Nantel Bergeron and Sara Billey restricted their attention to two rowed chute moves. For this case, their main theorem states that the poset defined by chute moves has a unique maximal element, namely

$$
D_{\text {top }}(w)=\left\{(c, j): c \leq \#\left\{i: i<w_{j}^{-1}, w_{i}>j\right\}\right\} .
$$

It is easy to see that considering general chute moves, the poset has also a unique minimal element, namely

$$
D_{b o t}(w)=\left\{(i, c): c \leq \#\left\{j: j>i, w_{j}<w_{i}\right\}\right\} .
$$

In the next section we will show a statement similar in spirit to the main theorem of Nantel Bergeron and Sara Billey for the more general chute moves defined above.

After generating and analysing some of these posets using Sage [14], see Figure 3 for an example, we became convinced that they should have much more structure:

Conjecture 2.8 The poset of reduced pipe dreams defined by (general) chute moves is in fact a lattice.

There is another natural way to transform one reduced pipe dream into another, originating in the concept of flipping a diagonal of a triangulation. Namely, consider an elbow joint in the pipe dream. Since any pair of pipes crosses at most once, there is at most one location where the pipes originating from the given elbow joint cross. If there is such a crossing, replace the elbow joint by a cross and the cross by an elbow joint. Clearly, the result is again a reduced pipe dream, associated to the same permutation.

It is believed (see Vincent Pilaud and Michel Pocchiola [11], Question 51) that the simplicial complex of multitriangulations can be realised as a polytope, in this case the graph of flips would be the graph of the polytope. Note that graph of chute moves is a subgraph of the graph of flips. Is Conjecture 2.8 related to the question of polytopality?

\section{Maximal Fillings of Moon Polyominoes and Pipe Dreams}

Consider a filling in $\mathcal{F}_{01}^{n e}(M, k)$. Replacing zeros with crosses, and all cells containing ones as well as all cells not in $M$ with elbow joints we clearly obtain a pipe dream. We will see in this section that it in fact reduced.

Even without that knowledge we can speak of chute moves applied to fillings in $\mathcal{F}_{01}^{n e}(M, k)$. However, a priori it is not clear under which conditions the result of such a move is again a filling in $\mathcal{F}_{01}^{n e}(M, k)$. In particular we have to deal with the fact that under this identification all cells outside $M$ are also filled with elbow joints corresponding to ones. Of course, to determine the set of north-east chains we have to consider the original filling and the boundary of $M$ and disregard elbow joints outside.

Similar to the article of Nantel Bergeron and Sara Billey we will also consider two special fillings $D_{b o t}(M, k)$ and $D_{t o p}(M, k)$. These will turn out to be the minimal and the maximal element in the poset having elements $\mathcal{F}_{01}^{\text {ne }}(M, k)$, where one filling is smaller than another if it can be obtained by applying chute moves to the latter.

Definition 3.1 Let $M$ be a moon polyomino and $k \geq 0$. Then $D_{\text {top }}(M, k) \in \mathcal{F}_{01}^{n e}(M, k)$ is obtained by putting ones into all cells that can be covered by any rectangle of size at most $k \times k$ which is completely contained in the moon polyomino and that touches the boundary of $M$ with its lower-left corner. 


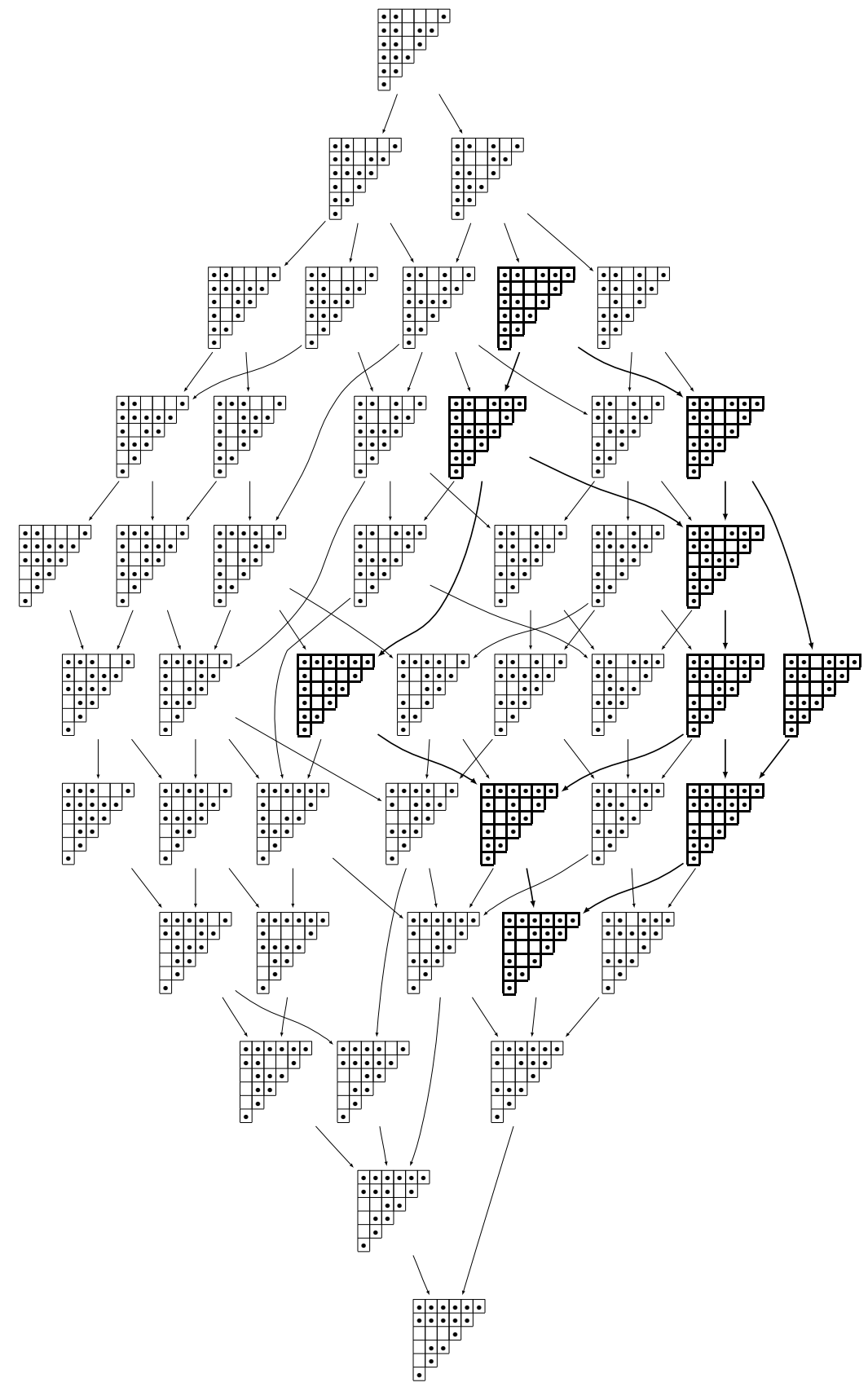

Fig. 3: the poset of reduced pipe dreams for the permutation $1,2,6,4,5,3$. The interval of 0 -1-fillings with $k=1$ of the moon polyomino $\square$ is shown in bold. 
Similarly, $D_{\text {bot }}(M, k) \in \mathcal{F}_{01}^{n e}(M, k)$ is obtained by putting ones into all cells that can be covered by any rectangle of size at most $k \times k$ which is completely contained in the moon polyomino and that touches the boundary of $M$ with its upper-right corner.
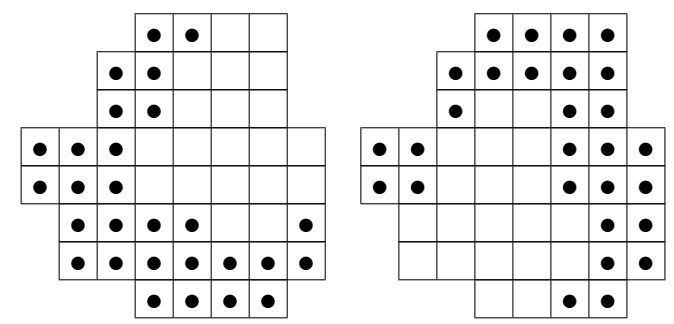

Fig. 4: The special fillings $D_{t o p}(M, k)$ and $D_{b o t}(M, k)$ for $k=2$ of a moon polyomino.

We can now state the main theorem of this article:

Theorem 3.2 Let $M$ be a moon polyomino and $k \geq 0$. The set $\mathcal{F}_{01}^{n e}(M, k, \mathbf{r})$ can be identified with the set of reduced pipe dreams $\mathcal{R C}(w(M, k), \mathbf{r})$ having all crosses inside of $M$ for some permutation depending only on $M$ and $k$ : replace zeros with crosses and all cells containing ones as well as all cells not in $M$ with elbow joints.

More precisely, the set $\mathcal{F}_{01}^{\text {ne }}(M, k)$ is an interval in the poset of reduced pipe dreams $\mathcal{R C}(w(M, k))$ with minimal element $D_{b o t}(M, k)$ and maximal element $D_{\text {top }}(M, k)$.

As already remarked in the introduction various versions of this theorem were independently proved by various authors by various methods. The most general version is due to Luis Serrano and Christian Stump [15, Theorem 2.6] who use properties of subword complexes and obtain additionally many properties of the simplicial complex of 0-1-fillings.

The advantage of our approach using chute moves is the demonstration of the property that $\mathcal{F}_{01}^{n e}(M, k)$ is in fact an interval in the bigger poset of reduced pipe dreams. In particular, if Conjecture 2.8 turns out to be true $\mathcal{F}_{01}^{n e}(M, k)$ is also a lattice. An illustration is given in Figure 3.

Let us first state a very basic property of chute moves as applied to fillings:

Lemma 3.3 Let $M$ be a moon polyomino. Chute moves and their inverses applied to a filling in $\mathcal{F}_{01}^{n e}(M, k)$ produce another filling in $\mathcal{F}_{01}^{\text {ne }}(M, k)$ whenever all zero entries remain in $M$.

Proof: We only have to check that chain lengths are preserved which is not hard.

Most of what remains of this section is devoted to prove that there is precisely one filling in $\mathcal{F}_{01}^{n e}(M, k)$ that does not admit a chute move such that the result is again in $\mathcal{F}_{01}^{n e}(M, k)$, namely $D_{b o t}(M, k)$, and precisely one filling that does not admit an inverse chute move with the same property, namely $D_{\text {top }}(M, k)$.

Although the strategy itself is actually very simple the details turn out to be quite delicate. Because of space constraints we omit the proofs in this extended abstract. However, we try to give an impression of the overall structure of the proof and state some of the auxiliary lemmas. Let us fix $k$, a moon polyomino $M$ and a maximal filling $D \in \mathcal{F}_{01}^{n e}(M, k)$ different from $D_{b o t}(M, k)$. We will then explicitly locate a chutable rectangle. Note that maximality of the filling will play a crucial role throughout. The first lemma 
is used to show that certain cells of the polyomino must be empty because otherwise the filling would contain a chain of length $k+1$ :

Lemma 3.4 (Chain induction) Consider a maximal filling of a moon polyomino. Let $\epsilon$ be an empty cell such that all cells below $\epsilon$ in the same column are empty too, except possibly those that are below the lowest cell of the column left of $\epsilon$. Assume that for each of these cells $\delta$ there is a maximal chain for $\delta$ strictly north-east of $\delta$. Then there is a maximal chain for $\epsilon$ strictly north-east of $\epsilon$.

Note that for the conclusion of Lemma 3.4 to hold we really have to assume that all cells below $\epsilon$ are empty: in the maximal filling for $k=1$

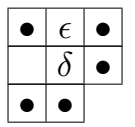

there is a maximal chain for $\delta$ north-east of $\delta$, but no maximal chain for $\epsilon$ north-east of $\epsilon$. The following example demonstrates that it is equally necessary that the filling is maximal:

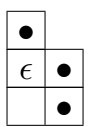

The next lemma parallels the main Lemma 3.6 in the article by Nantel Bergeron and Sara Billey [1]:

Lemma 3.5 Consider a maximal filling of a moon polyomino. Suppose that there is a cell $\gamma$ containing a 1 with an empty cell $\epsilon$ in the neighbouring cell to its right such that there are at least as many cells above $\gamma$ as above $\epsilon$. Then the filling contains a chutable rectangle.

Finally, the main statement follows from a careful analysis of fillings different from $D_{b o t}(M, k)$, repeatedly applying the previous lemmas to exclude obstructions to the existence of a chutable rectangle:

Theorem 3.6 Any maximal filling other than $D_{b o t}(M, k)$ admits a chute move such that the result is again a filling of $M$. Any maximal filling other than $D_{\text {top }}(M, k)$ admits an inverse chute move such that the result is again a filling of $M$.

Proof of Theorem 3.2; All pipe dreams in $\mathcal{R C}(w)$ contained in $M$ are maximal 0-1 fillings of $M$ since they can be generated by applying sequences of chute moves to $D_{t o p}(M, k)$.

Since we can apply chute moves to any maximal 0-1-filling of $M$ except $D_{b o t}(M, k)$ all such fillings arise in this fashion. (We have to remark here that in case the pipe dream associated to some filling would not be reduced, applying chute moves eventually exhibits that the filling was not maximal.) Together with Lemma 3.3 this implies that all fillings $F_{01}^{n e}(M, k)$ have the same associated permutation.

Note that, as a by-product, this procedure implies that all maximal 0-1-fillings of $M$ have the same number of entries equal to zero, i.e., the simplicial complex of fillings is pure.

\section{Applying the Edelman-Greene correspondence}

Using the identification described in the previous section, we can apply a correspondence due to Paul Edelman and Curtis Greene [2], that associates pairs of tableaux to reduced factorisations of permutations. This in turn will yield the desired bijective proof of Jakob Jonsson's result at least for stack polyominoes. 
The main result of this section was obtained for Ferrers shapes earlier by Luis Serrano and Christian Stump [15] using the same proof strategy. For stack polyominoes the description of the $P$-tableau is different, thus we believe it is useful to repeat the arguments here.

The following theorem is a collection of results from Paul Edelman and Curtis Greene [2], Richard Stanley [16], Alain Lascoux and Marcel-Paul Schützenberger [9], and describes properties of the EdelmanGreene correspondence:

Theorem 4.1 There is a bijection between pairs of words reduced factorisations of a permutation $w$ and pairs $(P, Q)$ of Young tableaux of the same shape, such that $P$ is column strict with reading word reduced equivalent to $w$, and $Q$ is standard. Moreover, if $w$ is vexillary, i.e., 2143-avoiding, the tableau $P$ is the same for all reduced factorisations of $w$.

It turns out that the permutations associated to moon polyominoes are indeed vexillary:

Proposition 4.2 For any moon-polyomino $M$ and any $k$, the permutation $w(M, k)$ is vexillary.

There are vexillary permutations which do not correspond to moon polyominoes. For example, the only two reduced pipe dreams for the permutation $4,2,5,1,3$ are as follows:
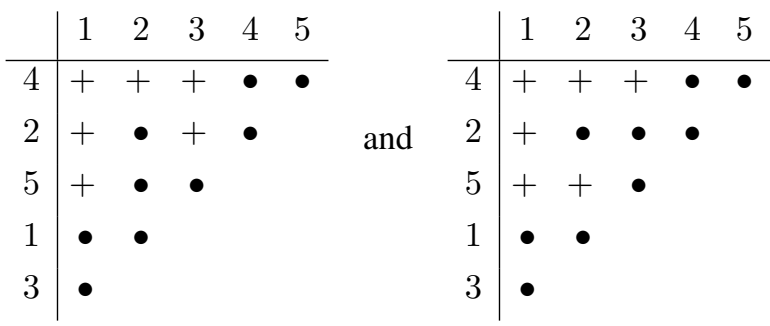

Proof: It is sufficient to prove the claim for $k=0$, since the empty cells in the filling $D_{t o p}(M, k)$ for any $k$ again form a moon polyomino. Thus, suppose that the permutation associated to $M$ is not vexillary. Then we have indices $i<j<k<\ell$ such that $w(j)<w(i)<w(\ell)<w(k)$. It follows that the pipes entering in columns $i$ and $j$ from above cross, and so do the two pipes entering in columns $k$ and $\ell$, and thus correspond to cells of the moon polyomino. Since any two cells in the moon polyomino can be connected by a path of neighbouring cells changing direction at most once, there is a third cell where either the pipes entering from $i$ and $\ell$ or from $j$ and $k$ cross, which is impossible.

Theorem 4.3 (for Ferrers shapes, Luis Serrano and Christian Stump [15]) For a stack polyomino $S$, consider the set $\mathcal{F}_{01}^{n e}(S, k, \mathbf{r})$. Let $\mu_{i}$ be the number of cells the $i^{\text {th }}$ row of $S$ is indented to the right, and suppose that $\mu_{1}=\cdots=\mu_{k}=\mu_{k+1}=0$.

Let $u$ be the word $1^{\mathbf{r}_{1}}, 2^{\mathbf{r}_{2}}, \ldots$ and let $v$ be the reduced factorisation of $w$ associated to a given pipe dream. Then the Edelman-Greene correspondence applied to the pair of words $(u, v)$ induces a bijection between $\mathcal{F}_{01}^{n e}(S, k, \mathbf{r})$ and the set of pairs $(P, Q)$ of Young tableaux satisfying the following conditions:

- the common shape of $P$ and $Q$ is the multiset of column heights of the empty cells in $D_{\text {top }}(S, k)$,

- the first row of $P$ equals $\left(k+1, k+2+\mu_{k+2}, k+3+\mu_{k+3}, \ldots\right)$, and the entries in columns are consecutive,

- $Q$ has type $\left\{1^{\mathbf{r}_{1}}, 2^{\mathbf{r}_{2}}, \ldots\right\}$, and entries in column $i$ are at most $i+k$. 
Thus, the common shape of $P$ and $Q$ encodes the row lengths of $S$, the entries of the first row of $P$ encode the left border of $S$, and the entries of $Q$ encode the filling.

In particular, this theorem implies an explicit bijection between the sets $\mathcal{F}_{01}^{n e}\left(S_{1}, k, \mathbf{r}\right)$ and $\mathcal{F}_{01}^{n e}\left(S_{2}, k, \mathbf{r}\right)$, given that the multisets of column heights of $S_{1}$ and $S_{2}$ coincide.

Curiously, the most natural generalisation of the above theorem to moon polyominoes is not true. Namely, one may be tempted to replace the condition on $Q$ by requiring that the entries of $Q$ are between $Q_{t o p}$ and $Q_{b o t}$ component-wise. However, this fails already for $k=1$ and the reverse Ferrers shape

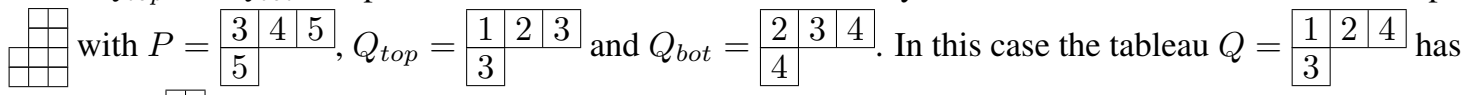
preimage $\because: 0$

One might hope to prove Conjecture 2.8 by applying the Edelman-Greene correspondence, and checking that the poset is a lattice on the tableaux. However, at least for the natural component-wise order on tableaux, the correspondence is not order preserving, not even for the case of Ferrers shapes.

Proof: In view of Proposition 4.2, to obtain the tableau $P$ it is enough to insert the reduced word given by the filling $D_{\text {top }}(S, k)$ using the Edelman-Greene correspondence, which is not hard for stack polyominoes.

It remains to prove that the entries in column $i$ of $Q$ are at most $i+k$ precisely if $(u, v)$ comes from a filling in $\mathcal{F}_{01}^{n e}(S, k)$. To this end, observe that the shape of the first $i$ columns of $P$ equals the shape of the tableau obtained after inserting the pair of words $\left(\left(u_{1}, u_{2}, \ldots, u_{\ell}\right),\left(v_{1}, v_{2}, \ldots, v_{\ell}\right)\right)$, where $\ell$ is such that $u_{\ell} \leq k+i$ and $u_{\ell+1}>k+i$.

Namely, this is the case if and only if the first $i+k+\mu_{i+k+1}$ positions of the permutation corresponding to $\left(v_{1}, v_{2}, \ldots, v_{\ell}\right)$ coincide with those of the permutation $w$ corresponding to $v$ itself, as can be seen by considering $D_{t o p}(w)$, whose empty cells form again a stack polyomino.

This in turn is equivalent to all letters $v_{m}$ being at least $k+i+1+\mu_{k+i+1}$ for $m>\ell$, i.e., whenever the corresponding empty cell of the filling occurs in a row below the $(i+k)^{\text {th }}$ of $S$, and thus, when it is inside $S$.

\section{Acknowledgements}

I am very grateful to my wife for encouraging me to write this note, and for her constant support throughout. I would also like to thank Thomas Lam and Richard Stanley for extremely fast replies concerning questions about Theorem 4.1 .

I would like to acknowledge that Christian Stump provided a preliminary version of [17]. Luis Serrano and Christian Stump informed me privately that they were able to prove that all $k$-fillings of Ferrers shapes yield the same permutation $w$, however, their ideas would not work for stack polyominoes. I was thus motivated to attempt the more general case.

\section{References}

[1] Nantel Bergeron and Sara Billey, RC-graphs and Schubert polynomials, Experiment. Math. 2 (1993), no. 4, 257-269, Available from: http://projecteuclid.org/getRecord?id=euclid. em/1048516036. MR 1281474 (95g:05107) 
[2] Paul Edelman and Curtis Greene, Balanced tableaux, Adv. in Math. 63 (1987), no. 1, 42-99, do i: 10.1016/0001-8708(87)90063-6. MR 871081 (88b:05012)

[3] Sergey Fomin and Anatol N. Kirillov, The Yang-Baxter equation, symmetric functions, and Schubert polynomials, Proceedings of the 5th Conference on Formal Power Series and Algebraic Combinatorics (Florence, 1993), vol. 153, 1996, pp. 123-143, doi:10.1016/0012-365X(95) 00132-G. MR 1394950 (98b:05101)

[4] __ Reduced words and plane partitions, J. Algebraic Combin. 6 (1997), no. 4, 311-319, doi: 10.1023/A:1008694825493. MR 1471891 (98m:05196)

[5] Jakob Jonsson, Generalized triangulations and diagonal-free subsets of stack polyominoes, Journal of Combinatorial Theory, Series A 112 (2005), no. 1, 117-142. MR MR2167478 (2006d:05011)

[6] Allen Knutson and Ezra Miller, Subword complexes in Coxeter groups, Adv. Math. 184 (2004), no. 1, 161-176, doi:10.1016/S0001-8708 (03) 00142-7, MR 2047852 (2005c:20066)

[7] __ Gröbner geometry of Schubert polynomials, Ann. of Math. (2) 161 (2005), no. 3, 1245 1318, doi:10.4007/annals.2005.161.1245. MR 2180402 (2006i:05177)

[8] Christian Krattenthaler, Growth diagrams, and increasing and decreasing chains in fillings of Ferrers shapes, Advances in Applied Mathematics 37 (2006), no. 3, 404-431, arXiv:math.CO/ 0510676. MR MR2261181 (2007h:05011)

[9] Alain Lascoux and Marcel-Paul Schützenberger, Structure de Hopf de l'anneau de cohomologie et de l'anneau de Grothendieck d'une variété de drapeaux, Comptes Rendus des Séances de l'Académie des Sciences. Série I. Mathématique 295 (1982), no. 11, 629-633. MR 686357 (84b:14030)

[10] Vincent Pilaud, Multitriangulations, pseudotriangulations et quelques problèmes de réalisation de polytopes, Ph.D. thesis, Université Paris Diderot Paris 7, 2010, arXiv:1009.1605 [math.CO].

[11] Vincent Pilaud and Michel Pocchiola, Multi-pseudotriangulations, 25th European Workshop on Computational Geometry, EuroCG'09, 2009, pp. 227-230, arXiv: http://2009.eurocg. org/abstracts.pdf

[12] Vincent Pilaud and Francisco Santos, Multitriangulations as complexes of star polygons, Discrete Comput. Geom. 41 (2009), no. 2, 284-317, doi:10.1007/s00454-008-9078-6. MR 2471876 (2009m:52007)

[13] Martin Rubey, Increasing and Decreasing Sequences in Fillings of Moon Polyominoes, Advances in Applied Mathematics (2011), (in press), arXiv:math.CO/0604140, doi:10.1016/j. aam.2009.11.013.

[14] The Sage-Combinat community, Sage-Combinat: enhancing sage as a toolbox for computer exploration in algebraic combinatorics, 2010, Available from: http: / combinat . sagemath.org.

[15] Luis Serrano and Christian Stump, Maximal fillings of moon polyominoes, simplicial complexes, and Schubert polynomials, Preprint (2010), arXiv:1009.4690 [math.CO] 
[16] Richard P. Stanley, On the number of reduced decompositions of elements of Coxeter groups, European Journal of Combinatorics 5 (1984), no. 4, 359-372. MR 782057 (86i:05011)

[17] Christian Stump, A new perspective on k-triangulations, Journal of Combinatorial Theory, Series A 118 (2011), no. 6, 1794 - 1800, arXiv:1009.4101 [math.CO], doi:10.1016/j.jcta. 2011.03 .001 .

[18] Alexander Woo, Catalan numbers and Schubert polynomials for $w=1(n+1) \ldots 2$, Preprint (2004), arXiv:math.co/0407160 
